Discrete Comput Geom 36:573-592 (2006)

DOI: $10.1007 / \mathrm{s} 00454-006-1266-7$

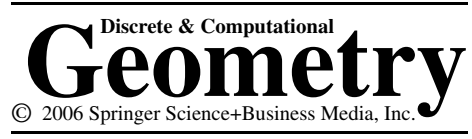

\title{
A Two-Dimensional Kinetic Triangulation with Near-Quadratic Topological Changes*
}

\author{
Pankaj K. Agarwal, ${ }^{1}$ Yusu Wang, ${ }^{2}$ and Hai $\mathrm{Yu}^{1}$ \\ ${ }^{1}$ Department of Computer Science, Duke University, \\ Box 90129, Durham, NC 27708, USA \\ \{pankaj,fishhai\}@cs.duke.edu \\ ${ }^{2}$ Department of Computer Science and Engineering, Ohio State University, \\ 2015 Neil Avenue, Columbus, OH 43210, USA \\ yusu@cse.ohio-state.edu
}

\begin{abstract}
A triangulation of a set $S$ of points in the plane is a subdivision of the convex hull of $S$ into triangles whose vertices are points of $S$. Given a set $S$ of $n$ points in $\mathbb{R}^{2}$, each moving independently, we wish to maintain a triangulation of $S$. The triangulation needs to be updated periodically as the points in $S$ move, so the goal is to maintain a triangulation with a small number of topological events, each being the insertion or deletion of an edge. We propose a kinetic data structure (KDS) that processes $n^{2} 2^{O(\sqrt{\log n \cdot \log \log n})}$ topological events with high probability if the trajectories of input points are algebraic curves of fixed degree. Each topological event can be processed in $O(\log n)$ time. This is the first known KDS for maintaining a triangulation that processes a near-quadratic number of topological events, and almost matches the $\Omega\left(n^{2}\right)$ lower bound [1]. The number of topological events can be reduced to $n k \cdot 2^{O(\sqrt{\log k \cdot \log \log n)}}$ if only $k$ of the points are moving.
\end{abstract}

\section{Introduction}

A triangulation of a set $S$ of points in $\mathbb{R}^{2}$ is a subdivision of the convex hull of $S$ into triangles whose vertices are points of $S$. Motivated by applications in several areas, including computer graphics, physical simulation, collision detection, and geographic information systems, triangulations have been widely studied [9], [12]. With the advancement

\footnotetext{
* This research was supported by NSF under Grants CCR-00-86013, EIA-98-70724, EIA-01-31905, CCR02-04118, and DEB-04-25465, by ARO Grants W911NF-04-1-0278 and DAAD19-03-1-0352, by a grant from the U.S.-Israel Binational Science Foundation. This work was done while the second author was a graduate student at Duke University.
} 
in technology, many applications, for instance, video games, virtual reality, dynamic simulations, and robotics, call for maintaining a triangulation as the points move. For example, the arbitrary Eulerian-Lagrangian method [11] provides a way to integrate the motion of fluids and solids within a moving finite-element mesh. The time axis is discretized and the mesh vertices are moved between each time step so as to respect the interfaces between the different media. However, numerical problems arise when the mesh becomes too distorted, and the mesh generated depends on the discretization of time. Another approach to build more adaptive triangulations for such problems is to work in the space-time domain [13].

Given a set $S$ of points in $\mathbb{R}^{2}$, each moving independently, we wish to maintain a triangulation of $S$. As the points in $S$ move, any fixed triangulation of $S$ also deforms continuously. However, a triangulation computed for the initial configuration cannot be guaranteed to remain valid all the time. Therefore, it becomes necessary to update a triangulation over time by deleting some of the existing edges and inserting some other edges. We refer to each such insertion or deletion in the triangulation as a topological event. The topological events occur only at discrete time instances. In this paper we study how to maintain a triangulation so that the number of topological events is near-quadratic.

Related Work. Since Atallah's seminal paper [7] on kinetic geometry, much work has been devoted to this area due to its importance in both theory and applications of computational geometry; see [3], [5], and [14] for reviews on kinetic geometric algorithms and data structures. The early work on kinetic geometry mostly focused on bounding the number of combinatorial or topological changes in various geometric structures as the input objects move. Basch et al. [8] introduced a general framework, the so-called kinetic data structure (KDS), for maintaining a discrete attribute of objects in predictable motion. Their approach to maintain a given attribute $A(t)$ for a continuously changing scene $S(t)$ is as follows: at a given time $t$, we create a proof of correctness of the attribute based on elementary tests called certificates. For each certificate, we compute the time at which it fails and put it in a global event queue. As the attribute cannot change while all tests remain valid, it is unnecessary to perform any computation until the first certificate fails. When a certificate fails, the discrete attribute is updated if it needs to be, and a new proof of correctness is constructed by making certain modifications to the previous proof of correctness. Their approach led to efficient algorithms for several kinetic problems [14].

In the context of triangulation, a longstanding open problem is to bound the number of topological events in the Delaunay triangulation of a set of moving points in $\mathbb{R}^{2}$. The best known upper bound is near-cubic if trajectories of input points are algebraic curves of fixed degree; the bound is cubic if each point moves with unit speed in a fixed direction [6]. Although it is conjectured that the number of topological changes is $O\left(n^{2}\right)$, no such bound is known even for maintaining an arbitrary triangulation of a set of moving points in $\mathbb{R}^{2}$. Agarwal et al. [2] described a scheme for maintaining a triangulation of a set of points that incurs roughly $n^{7 / 3}$ topological changes if the points are moving linearly. Chew [10] proved that the Delaunay triangulation of $S$ under $L_{1}$-metric changes $O\left(n^{2} \alpha(n)\right)$ times, where $\alpha(n)$ is the inverse Ackermann function; however, the Delaunay triangulation under $L_{1}$-metric is not necessarily a triangulation of the convex hull of the point set. 
Agarwal et al. [1] showed that the convex hull of a moving point set may change $\Theta\left(n^{2}\right)$ times if the points are moving linearly; this result immediately implies a lower bound $\Omega\left(n^{2}\right)$ on the number of topological changes to any triangulation. This lower bound on the number of topological events holds even if a linear number of Steiner points are allowed [4].

Our Results. Let $S=\left\{p_{1}, \ldots, p_{n}\right\}$ be a set of points moving in $\mathbb{R}^{2}$. Let $p_{i}(t)=$ $\left(x_{i}(t), y_{i}(t)\right)$ denote the position of $p_{i}$ at time $t$, and let $S(t)$ denote the configuration of $S$ at time $t$. We assume that $x_{i}(\cdot), y_{i}(\cdot)$ are polynomials of fixed degree. We describe a KDS for maintaining a triangulation of $S(t)$ that processes $n^{2} 2^{O(\sqrt{\log n \cdot \log \log n})}$ topological events with high probability, each of which is insertion or deletion of an edge. Each topological event can be processed in $O(\log n)$ time. This is the first KDS for maintaining a triangulation that processes near-quadratic topological events. The number of events can be reduced to $n k \cdot 2^{O(\sqrt{\log k \cdot \log \log n})}$ if only $k$ of the points are moving.

Our algorithm relies on a randomized hierarchical scheme. We first describe the so-called fan triangulation (Section 2), and then introduce the notion of constrained fan triangulation with respect to a planar subdivision (Section 3 ). We choose a random sample $R \subseteq S$, compute a triangulation of $R$ recursively, and then compute the constrained fan triangulation of $S$ with respect to the triangulation computed for $R$ (Section 4). We analyze the events in constrained fan triangulation and show that if $R$ is a random subset of appropriate size, the total number of events is near-quadratic (Section 5).

\section{Fan Triangulation}

Let $S=\left\{p_{1}, \ldots, p_{n}\right\}$ be a set of $n$ (stationary) points in $\mathbb{R}^{2}$, sorted in non-increasing order of their $y$-coordinates, i.e., $y\left(p_{1}\right) \geq y\left(p_{2}\right) \geq \cdots \geq y\left(p_{n}\right)$. For a point $q \in \mathbb{R}^{2}$, let $S_{q}=\left\{p_{i} \in S \mid y\left(p_{i}\right)>y(q)\right\}$. Denote by $\mathcal{V}(q) \subseteq S$ the set of points on $\partial \operatorname{conv}\left(S_{q}\right)$ that are visible from $q$, i.e., $p_{i} \in \mathcal{V}(q)$ if the relative interior of the segment $q p_{i}$ does not intersect $\operatorname{conv}\left(S_{q}\right)$. Furthermore, let $\rho(q)$ denote the point from $\mathcal{V}(q)$ such that the oriented line $\overrightarrow{q \rho(q)}$ is the left tangent of $\operatorname{conv}\left(S_{q}\right)$, and let $\gamma(q)$ denote the point from $\mathcal{V}(q)$ such that the oriented line $\overrightarrow{q \gamma(q)}$ is the right tangent of $\operatorname{conv}\left(S_{q}\right)$. Obviously, $\mathcal{V}(q)$ is the subset of vertices on $\partial \operatorname{conv}\left(S_{q}\right)$ lying between $\rho(q)$ and $\gamma(q)$, assuming that the vertices are ordered in counterclockwise direction.

The fan triangulation of $S$ is constructed by sweeping a horizontal line $h$ from $y=$ $+\infty$ to $y=-\infty$. At any time the algorithm maintains the fan triangulation of points from $S$ that lie above $h$. It updates the triangulation when the sweep line crosses a point $p_{i} \in S$ by adding the edges $p_{i} p_{j}$ for all $p_{j} \in \mathcal{V}\left(p_{i}\right)$; see Fig. 1 . The triangulation at the end of the sweep is the fan triangulation of $S$, which we denote as $\mathbb{F}(S)$.

We classify the edges of $\mathbb{F}(S)$ incident upon a point $p_{i} \in S$ into two classes:

(i) Up edges: edges $p_{i} p_{j}$ so that $j<i ; p_{j}$ is also referred to as an up neighbor of $p_{i}$.

(ii) Down edges: edges $p_{i} p_{j}$ so that $j>i ; p_{j}$ is also called a down neighbor of $p_{i}$. Furthermore, if $p_{i}=\rho\left(p_{j}\right)$ or $p_{i}=\gamma\left(p_{j}\right)$, then edge $p_{i} p_{j}$ is referred to as a convex edge; otherwise, it is a reflex edge. 


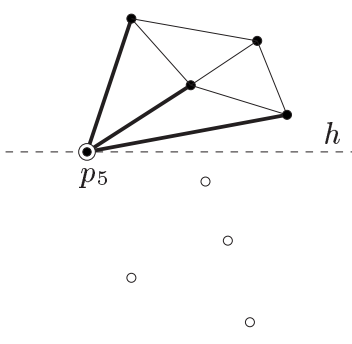

(a)

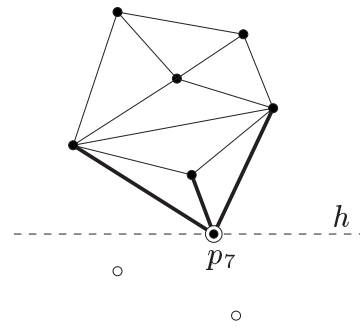

(b)

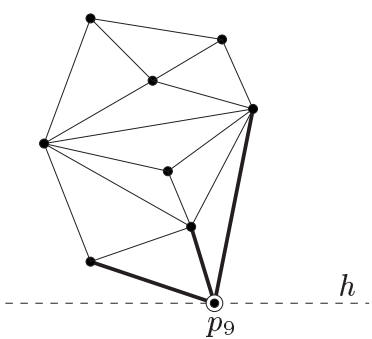

(c)

Fig. 1. Construction of fan triangulation at various stages - the point denoted by double circle is being inserted, and the thick edges are added. The points are ordered from top to bottom by their indices.

The following properties of $\mathbb{F}(S)$ are straightforward to prove:

(F1) For $1 \leq i<n, p_{i} p_{i+1}$ is an edge of $\mathbb{F}(S)$.

(F2) For each $p_{i}$, at most one of its down edges is a reflex edge. Indeed if $p_{i} p_{j}$ is a reflex edge of $p_{i}$, then $p_{i}$ lies in the interior of $\operatorname{conv}\left(S_{p_{j+1}}\right)$, and therefore there is no down edge $p_{i} p_{k}$, for $k>j$. Let the edges $p_{i} p_{j_{1}}, p_{i} p_{j_{2}}, \ldots, p_{i} p_{j_{k}}$ be the sequence of the down edges incident upon $p_{i}$ sorted in the clockwise direction. Then either $j_{1}<\cdots<j_{k}$ and $p_{i}=\rho\left(p_{j_{l}}\right)$ for $l<k\left(l \leq k\right.$ if $p_{i} p_{j_{k}}$ is not a reflex edge, e.g., $p_{5}$ in Fig. 1(c)), or $j_{1}>\cdots>j_{k}$ and $p_{i}=\gamma\left(p_{j_{l}}\right)$ for $l<k$ ( $l \leq k$ if $p_{i} p_{j_{k}}$ is not a reflex edge, e.g., $p_{4}$ in Fig. 1(c)).

Now suppose we are given a set of moving points $S(t)=\left\{p_{1}(t), p_{2}(t), \ldots, p_{n}(t)\right\}$ in the plane, and we wish to maintain $\mathbb{F}(S(t))$ for any $t \in \mathbb{R}$. As the points from $S$ move, $\mathbb{F}(S(t))$ deforms continuously. However, the topology of $\mathbb{F}(S(t))$ changes only at discrete times, which we refer to as events. Note the difference between events and topological events: an event may cause multiple topological events to the triangulation. As observed in [2], there are two types of events.

Ordering Event. The $y$-coordinates of two points $p_{i}$ and $p_{j}$ become equal at time $t_{0}$. Assume that $y\left(p_{i}\left(t_{0}^{-}\right)\right)>y\left(p_{j}\left(t_{0}^{-}\right)\right)$and $y\left(p_{i}\left(t_{0}^{+}\right)\right)<y\left(p_{j}\left(t_{0}^{+}\right)\right)$, where $t_{0}^{-}$is the time immediately before $t_{0}$ and $t_{0}^{+}$is the time immediately after $t_{0}$. By property $(\mathrm{F} 1), p_{i}\left(t_{0}^{-}\right) p_{j}\left(t_{0}^{-}\right)$ and $p_{j}\left(t_{0}^{+}\right) p_{i}\left(t_{0}^{+}\right)$are present in $\mathbb{F}\left(S\left(t_{0}^{-}\right)\right)$and $\mathbb{F}\left(S\left(t_{0}^{+}\right)\right)$, respectively. In fact, both of them are necessarily convex edges; assume $p_{i}\left(t_{0}^{-}\right)=\rho\left(p_{j}\left(t_{0}^{-}\right)\right)$and $p_{j}\left(t_{0}^{+}\right)=\gamma\left(p_{i}\left(t_{0}^{+}\right)\right)$. Let $p_{k_{1}}, p_{k_{2}}, \ldots, p_{k_{u}}$ be the sequence of vertices in $\mathcal{V}_{p_{i} p_{j}}\left(t_{0}\right)=\mathcal{V}\left(p_{i}\left(t_{0}^{-}\right)\right) \cap \mathcal{V}\left(p_{j}\left(t_{0}^{+}\right)\right)$ordered in the counterclockwise direction along $\partial \operatorname{conv}\left(S_{p_{i}}\left(t_{0}\right)\right)$. Obviously, at time $t_{0}^{-}$, $p_{k_{l}} p_{i}$, for all $1 \leq l \leq u$, and $p_{k_{u}} p_{j}$ are edges in $\mathbb{F}\left(S\left(t_{0}^{-}\right)\right)$, while at time $t_{0}^{+}, p_{k_{l}} p_{j}$, for all $1 \leq l \leq u$, and $p_{k_{1}} p_{i}$ are edges in $\mathbb{F}\left(S\left(t_{0}^{+}\right)\right)$. So to update the triangulation, we delete the edges $p_{k_{2}} p_{i}, \ldots, p_{k_{u}} p_{i}$ and insert the edges $p_{k_{1}} p_{j}, \ldots, p_{k_{u-1}} p_{j}$ to obtain $\mathbb{F}\left(S\left(t_{0}^{+}\right)\right)$. See Fig. 2(a). Hence, an ordering event induces $O\left(\left|\mathcal{V}_{p_{i} p_{j}}\left(t_{0}\right)\right|\right)$ topological events.

Visibility Event. For a point $p_{j} \in S$, either $\rho\left(p_{j}\right)$ or $\gamma\left(p_{j}\right)$ changes at time $t_{0}$. Suppose that $p_{i}\left(t_{0}^{-}\right)=\rho\left(p_{j}\left(t_{0}^{-}\right)\right)$and $p_{k}\left(t_{0}^{+}\right)=\rho\left(p_{j}\left(t_{0}^{+}\right)\right)$with $y\left(p_{k}\left(t_{0}\right)\right)>y\left(p_{i}\left(t_{0}\right)\right)$; the other cases are symmetric. Then $p_{i}\left(t_{0}\right), p_{j}\left(t_{0}\right)$, and $p_{k}\left(t_{0}\right)$ are collinear. Furthermore, among 

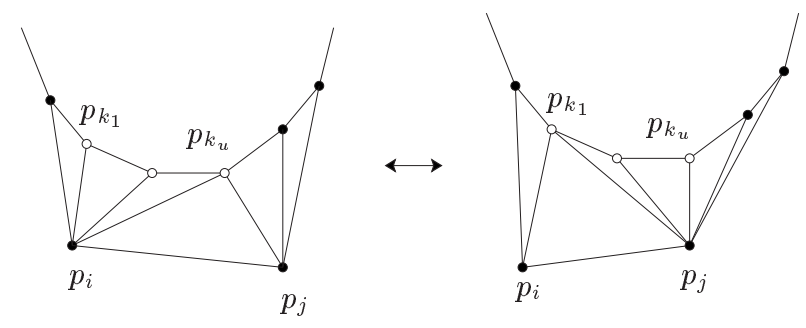

(a)
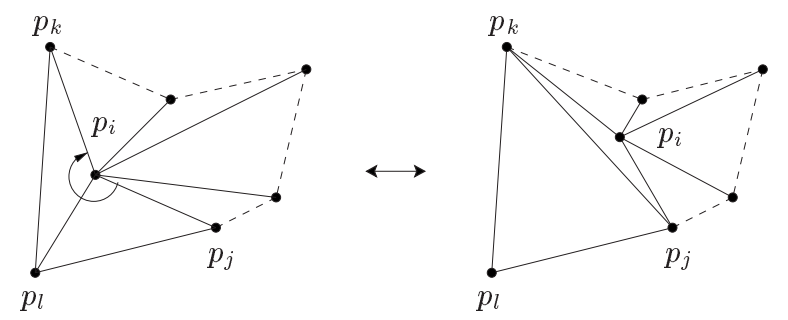

(b)

Fig. 2. (a) Ordering event. Points denoted by hollow circles are from $\mathcal{V}_{p_{i} p_{j}}\left(t_{0}\right)$. (b) Visibility event.

all the convex edges incident upon $p_{i}$ at $t_{0}^{-}, p_{i} p_{j}$ is the leftmost edge. It then follows from property $(\mathrm{F} 2)$ of the fan triangulation that there is at most one edge (the reflex edge), say $p_{i} p_{l}$, between $p_{i} p_{j}$ and $p_{i} p_{k}$ in clockwise order around $p_{i}$. If $p_{i} p_{l}$ does not exist, then $p_{i}, p_{j}$, and $p_{k}$ are collinear on $\partial \operatorname{conv}\left(S\left(t_{0}\right)\right)$. To update the fan triangulation, we delete the edge $p_{i} p_{l}$ (if it exists) and insert the edge $p_{j} p_{k}$ (Fig. 2(b)). Each visibility event induces $O(1)$ topological events.

In order to detect the above events, we maintain three families of certificates in a global priority queue:

(i) For each edge $p_{i} p_{j} \in \mathbb{F}(S(t))$, the next time $t_{0}$ at which $y\left(p_{i}\left(t_{0}\right)\right)=y\left(p_{j}\left(t_{0}\right)\right)$.

(ii) For each triangle $p_{i} p_{j} p_{k} \in \mathbb{F}(S(t))$, with $y\left(p_{j}\right)<y\left(p_{i}\right)<y\left(p_{k}\right)$, the next time $t_{0}$ at which $p_{i}\left(t_{0}\right) \in p_{j}\left(t_{0}\right) p_{k}\left(t_{0}\right)$.

(iii) For each point $p_{i}$, let $p_{j}$ be the point with the minimum $y$-coordinate so that $p_{i}=$ $\rho\left(p_{j}\right)$ (resp. $\left.p_{i}=\gamma\left(p_{j}\right)\right)$ if it exists. We add the time $t_{0}$ at which $p_{j}\left(t_{0}\right), p_{i}\left(t_{0}\right)$, and $\rho\left(p_{i}\left(t_{0}\right)\right)$ (resp. $\left.\gamma\left(p_{i}\left(t_{0}\right)\right)\right)$ become collinear.

By our above discussion, it is easy to verify that these certificates detect all events. Moreover, each topological event can be processed in $O(\log n)$ time, including the time spent in updating the global event queue.

\section{Constrained Fan Triangulation}

In this section we introduce the notion of constrained fan triangulation and show how to maintain it under motion. As earlier, let $S=\left\{p_{1}, \ldots, p_{n}\right\}$ be a set of $n$ (stationary) 

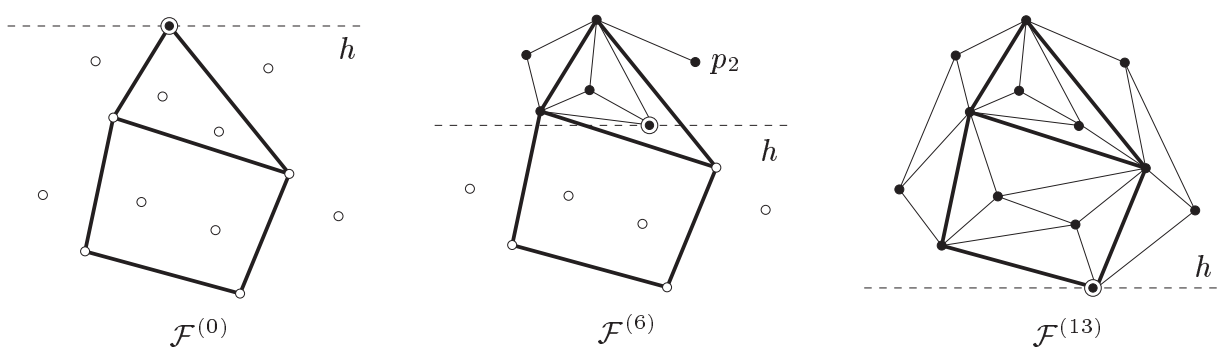

Fig. 3. Constructing constrained fan triangulation with respect to $\Pi$ (thick edges) at various stages.

points in $\mathbb{R}^{2}$ sorted in non-increasing order of their $y$-coordinates, and let $\Pi$ be a set of segments with pairwise-disjoint interiors whose endpoints lie in $S$. We are mostly interested in the case in which $\Pi$ is a triangulation of a subset $R \subseteq S$, but we give the definition for the general setting.

We construct the constrained fan triangulation of $S$ by sweeping a horizontal line $h$ from $y=+\infty$ to $y=-\infty$. Let $\mathbb{F}^{(0)}=\Pi$, and let $\mathbb{F}^{(i-1)}$ be the partial triangulation computed after the sweep line has processed $p_{i-1}$. If the interior of segment $p_{i} p_{j}$, for $j<i$, does not intersect $\mathbb{F}^{(i-1)}$, then $p_{i} p_{j}$ is called visible with respect to $\mathbb{F}^{(i-1)}$. Define $\mathcal{V}\left(p_{i}\right), \rho\left(p_{i}\right)$, and $\gamma\left(p_{i}\right)$ similarly as before, under the modified concept of visibility (with respect to $\mathbb{F}^{(i-1)}$ ); note that $\rho\left(p_{i}\right)$ and $\gamma\left(p_{i}\right)$ depend on $\Pi$. When the sweep line crosses $p_{i}$, we compute $\mathbb{F}^{(i)}$ by adding the edges $p_{i} p_{j}$ for all vertices $p_{j}$ in $\mathcal{V}\left(p_{i}\right)$. See Fig. 3. Note that unlike the sweeping process to construct the fan triangulation, $\mathbb{F}^{(i)}$ is not necessarily a constrained fan triangulation of the already swept points. In fact, $\mathbb{F}^{(i)}$ might not even be a triangulation; it is possible that only one point from $p_{1}, \ldots, p_{i-1}$ is visible from $p_{i}$ due to the constraint $\Pi$, in which case we add only one edge to $\mathbb{F}^{(i)}$, and this "dangling" edge of $\mathbb{F}^{(i)}$ will become part of a triangle at a later stage; see the edge incident upon $p_{2}$ in $\mathbb{F}^{(6)}$ of Fig. 3. The final triangulation $\mathbb{F}^{(n)}$ is the constrained fan triangulation of $S$ (with respect to $\Pi$ ), denoted by $\mathbb{F}(S, \Pi)$.

Observe that if $\Pi=\partial \operatorname{conv}(S)$, then $\mathbb{F}(S, \Pi)=\mathbb{F}(S)$. If $\Pi$ is a triangulation of a subset $R \subseteq S$, then $\Pi$ partitions $S$ into various subsets: $S_{\Delta}=S \cap \Delta$ (including vertices of $\Delta$ ), where $\Delta \in \Pi$ is a triangle or $\Delta=\Sigma$ is the exterior of the convex hull of $R$. By the above observation, the constrained fan triangulation $\mathbb{F}(S, \Pi)$ restricted to $S_{\Delta}$ is the same as $\mathbb{F}\left(S_{\Delta}\right)$ for $\Delta \in \Pi$. Hence, $\mathbb{F}(S, \Pi)$ can be computed by constructing independently $\mathbb{F}\left(S_{\Delta}\right)$ for each triangle $\Delta \in \Pi$ and constructing $\mathbb{F}\left(S_{\Sigma}, \partial \operatorname{conv}(R)\right)$ within the exterior of $\operatorname{conv}(R)$, i.e., the constrained fan triangulation of $S_{\Sigma}$ with respect to the boundary of $\operatorname{conv}(R)$.

The following properties of constrained fan triangulation are generalizations of $(\mathrm{F} 1)$ and (F2):

(C1) For $j<i$, if $p_{j}$ is the lowest vertex visible from $p_{i}$, then $p_{j} p_{i}$ is an edge in $\mathbb{F}(S, \Pi)$.

(C2) If $p_{i} p_{j_{1}}, \ldots, p_{i} p_{j_{k}}$ are the down edges incident upon $p_{i}$ sorted in clockwise direction so that no edges of $\Pi$ lie between them, then either $j_{1}<\cdots<j_{k}$ and $p_{i}=\rho\left(p_{j_{l}}\right)$ for $l<k\left(l \leq k\right.$ if $p_{i} p_{j_{k}}$ is not a reflex edge $)$, or $j_{1}>\cdots>j_{k}$ and $p_{i}=\gamma\left(p_{j_{l}}\right)$ for $l<k\left(l \leq k\right.$ if $p_{i} p_{j_{k}}$ is not a reflex edge). 
Next, we describe how to maintain $\mathbb{F}(S, \Pi)$ as the points in $S$ move. For the time being, we assume that $\Pi$ is a triangulation of a subset $R \subseteq S$ and that motion is such that the topology of $\Pi$ does not change and remains a valid triangulation of $R$ throughout the motion. In addition to ordering and visibility events, a new type of event, called the crossing event, arises when a point of $S \backslash R$ crosses an edge of $\Pi$. In the following we discuss each of them.

Ordering Event. There are two points $p_{i}, p_{j} \in S$ so that (i) $p_{i}\left(t_{0}\right)$ is visible from $p_{j}\left(t_{0}\right)$ with respect to $\Pi$, and (ii) $y\left(p_{i}\left(t_{0}\right)\right)=y\left(p_{j}\left(t_{0}\right)\right)$. An ordering event is processed in the same way as in Section 2. If $p_{i}\left(t_{0}\right)$ is not visible from $p_{j}\left(t_{0}\right)$, then $\mathbb{F}(S, \Pi)$ does not change at $t_{0}$. Note that the visibility relation between two points can change only by a crossing or a visibility event described below.

Visibility Event. For a point $p_{i} \in S$, either $\rho\left(p_{i}\right)$ or $\gamma\left(p_{i}\right)$ changes at time $t_{0}$, and $p_{i}$ does not cross any edge of $\Pi$ during $\left[t_{0}^{-}, t_{0}^{+}\right]$. We process this event in the same way as in Section 2. Note that $\rho\left(p_{i}\right)$ or $\gamma\left(p_{i}\right)$ could also change as $p_{i}$ crosses some edge from $\Pi$, which will be covered by the crossing event below.

Crossing Event. A point $p_{i}$ crosses an edge $p_{j} p_{k}$ of $\Pi$ at time $t_{0}$; assume $y\left(p_{k}\left(t_{0}\right)\right)>$ $y\left(p_{j}\left(t_{0}\right)\right)$. Recall that $\Pi$ is a triangulation of a subset of $S$. $\Pi$ partitions $\mathbb{R}^{2}$ into several connected components, each region being either a triangle of $\Pi$ or the exterior of conv $(R)$. Suppose $p_{i}$ moves from the region $\Delta^{-}$to $\Delta^{+}$. Let $S_{\Delta^{-}}$(resp. $S_{\Delta^{+}}$) be the subset of points of $S$ in $\Delta^{-}$(resp. $\Delta^{+}$) at $t_{0}^{-}$. Then the crossing event corresponds to deleting $p_{i}$ from $S_{\Delta^{-}}$, inserting it into $S_{\Delta^{+}}$, and updating the triangulations in $\Delta^{-}$and $\Delta^{+}$. First, we consider $\Delta^{-}$.

Given an arbitrary point $q \in S$, the star of $q$, denoted by $\operatorname{St}(q)$, is the union of triangles adjacent to $q$. $\operatorname{St}(q)$ is a star-shaped polygon with $q$ in its kernel—every point in $\operatorname{St}(q)$ is visible from $q$. The link of $q$, denoted by $\operatorname{Lk}(q)$, is defined as $\partial \operatorname{St}(q) . \operatorname{Lk}(q)$ is a closed polygonal curve. If $q \in \partial \operatorname{conv}(S)$, then $q \in \operatorname{Lk}(q)$; otherwise $q$ lies in the interior of $\operatorname{St}(q)$. Given the fan triangulation of $S, \operatorname{Lk}(q)$ consists of a convex chain, corresponding to the up neighbors of $q$, a $y$-monotone polygonal chain, corresponding to the down neighbors of $q$, and one more edge that connects the lowest neighbor of $q$ to an up neighbor of $q$ (or to $q$ if $q \in \partial \operatorname{conv}(S)$ ); see Fig. 4(a).

If $p_{i} \in \partial \operatorname{conv}(S)$ at time $t_{0}^{-}$(in which case $\Delta^{-}$represents the exterior of $\operatorname{conv}(R)$ ), we can simply remove edges $p_{i} p_{k}$ and $p_{i} p_{j}$ from $\mathbb{F}\left(S\left(t_{0}^{-}\right), \Pi\right)$. We now assume that $p_{i}\left(t_{0}^{-}\right) \notin \partial \operatorname{conv}\left(S\left(t_{0}^{-}\right)\right)$, implying that $p_{i}\left(t_{0}^{-}\right) \notin \operatorname{Lk}\left(p_{i}\left(t_{0}^{-}\right)\right)$.

Lemma 3.1. Within $\Delta^{-}$, any edge from $\mathbb{F}\left(S\left(t_{0}^{-}\right), \Pi\right)$ not incident upon $p_{i}$ at time $t_{0}^{-}$ is present in $\mathbb{F}(S(t), \Pi)$ for all $t \in\left[t_{0}^{-}, t_{0}^{+}\right]$.

The above lemma is straightforward, as any two points that are previously visible to each other within $\Delta^{-}$will remain visible after $p_{i}$ moves out of $\Delta^{-}$. In view of Lemma 3.1, we delete the edges incident upon $p_{i}$ at time $t_{0}^{-}$and re-triangulate within $\operatorname{Lk}\left(p_{i}\left(t_{0}^{-}\right)\right)$. The portion of the triangulation on and outside $\operatorname{Lk}\left(p_{i}\left(t_{0}^{-}\right)\right)$remains unchanged. We re-triangulate within $\operatorname{Lk}\left(p_{i}\left(t_{0}^{-}\right)\right)$as follows (Fig. 4). Let $Q=$ $\left\langle p_{k}=q_{0}, q_{1}, \ldots, q_{w}, q_{w+1}, \ldots, q_{u}=p_{j}\right\rangle$ be the sequence of vertices on $\operatorname{Lk}\left(p_{i}\right)$, where 


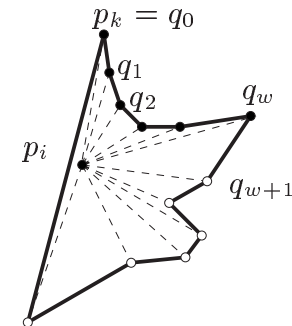

$p_{j}=q_{u}$

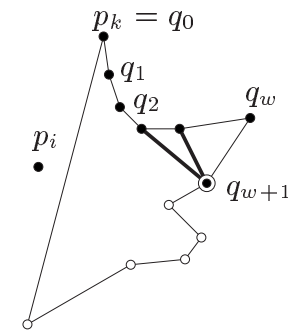

$p_{j}=q_{u}$ (a)

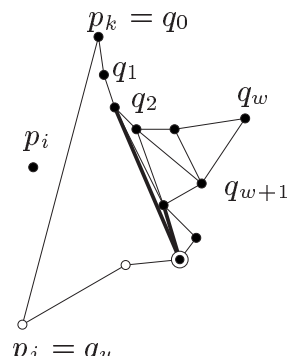

(c)

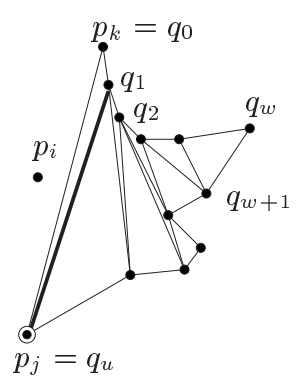

(d)

Fig. 4. Re-triangulation of $\operatorname{St}\left(p_{i}\left(t_{0}^{-}\right)\right)$after deleting $p_{i}$. The thick polygonal chain in (a) is $\operatorname{Lk}\left(p_{i}\left(t_{0}^{-}\right)\right)$.

$q_{0}, q_{1}, \ldots, q_{w}$ is the convex chain formed by the up neighbors of $p_{i}$, and $y\left(p_{i}\right)>$ $y\left(q_{w+1}\right)>y\left(q_{w+2}\right)>\cdots>y\left(q_{u}\right)$. Since $q_{0}, \cdots, q_{w}$ are already in convex position, we only visit $q_{w+1}, \ldots, q_{u}$ in order. Without loss of generality, assume that $p_{i}$ lies to the right of the edge $p_{k} p_{j}$ at time $t_{0}^{-}$, i.e., $p_{i}$ crosses $p_{k} p_{j}$ from right to left; the other case can be handled symmetrically. Suppose we have processed $q_{w+1}, \ldots, q_{z-1}$, i.e., added the new edges incident upon them. Letting $\rho^{(i)}(\cdot)=\rho\left(\rho^{(i-1)}(\cdot)\right)$, we maintain a subsequence of $Q$,

$$
\Phi^{(z-1)}=\left\langle q_{z-1}=\rho^{(0)}\left(q_{z-1}\right), \rho\left(q_{z-1}\right), \rho^{(2)}\left(q_{z-1}\right), \rho^{(3)}\left(q_{z-1}\right), \ldots\right\rangle,
$$

i.e., the vertices that appear on the left boundary of the convex hull of $q_{0}, \ldots, q_{z-1}$. If the vertices $q_{z-1}, \rho\left(q_{z-1}\right), \ldots, \rho^{(l)}\left(q_{z-1}\right)$ are visible from $q_{z}$, we then add the edges $q_{z} q_{z-1}, q_{z} \rho\left(q_{z-1}\right), \ldots, q_{z} \rho^{(l)}\left(q_{z-1}\right)$, delete $q_{z-1}, \ldots, \rho^{(l-1)}\left(q_{z-1}\right)$ from the sequence, set

$$
\Phi^{(z)}=\left\langle q_{z}, \rho\left(q_{z}\right)=\rho^{(l)}\left(q_{z-1}\right), \rho^{(l+1)}\left(q_{z-1}\right), \ldots\right\rangle,
$$

and repeat this process for $q_{z+1}$ unless $z=u$.

Next, we describe how to insert $p_{i}$ into $\Delta^{+}$and construct $\mathbb{F}\left(S_{\Delta^{+}} \cup\left\{p_{i}\right\}\right)$ at time $t_{0}^{+}$ from $\mathbb{F}\left(S_{\Delta^{+}}\right)$. Roughly speaking, we need to do the opposite of what we did in $\Delta^{-}$. That is, we identify $\operatorname{Lk}\left(p_{i}\left(t_{0}^{+}\right)\right)$in $\mathbb{F}\left(S_{\Delta^{+}} \cup\left\{p_{i}\right\}\right)$, delete the edges of $\mathbb{F}\left(S_{\Delta^{+}}\right)$that lie within 


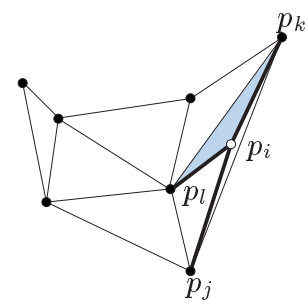

(a)

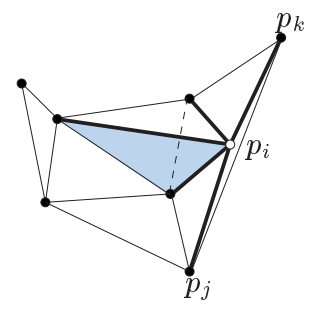

(c)

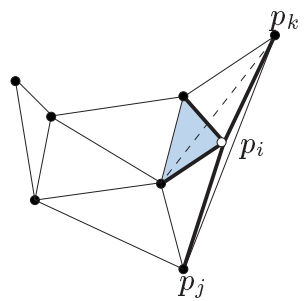

(b)

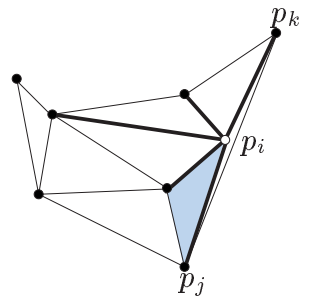

(d)

Fig. 5. Updating $\mathbb{F}\left(S_{\Delta^{+}}\right)$after inserting $p_{i}$ - the shaded triangle is being processed, and the dashed edge was flipped.

the polygon formed by $\operatorname{Lk}\left(p_{i}\left(t_{0}^{+}\right)\right)$, and connect $p_{i}$ to all the vertices on $\operatorname{Lk}\left(p_{i}\left(t_{0}^{+}\right)\right)$to form $\operatorname{St}\left(p_{i}\left(t_{0}^{+}\right)\right)$. We assume that $\Delta^{+}$lies to the left of $p_{k} p_{j}$. The following procedure performs these steps simultaneously.

Let $p_{l}$ be the vertex in $\Delta^{+}$adjacent to the edge $p_{j} p_{k}$ at time $t_{0}^{-}$. If $p_{l}$ does not exist, then $p_{j} p_{k}$ is an edge of $\operatorname{conv}(S)$, and $p_{i}$ becomes a vertex of $\operatorname{conv}(S)$, in which case we simply add the triangle $p_{i} p_{j} p_{k}$. We thus assume that $p_{l}$ exists. We add the edges $p_{i} p_{j}, p_{i} p_{k}$, and $p_{i} p_{l}$. We maintain a stack $\mathcal{S}$ of triangles. Initially, we push $p_{i} p_{l} p_{j}$ and $p_{i} p_{k} p_{l}$ to $\mathcal{S}$ (with the latter being on the top of $\mathcal{S}$ ). We perform the following procedure until $\mathcal{S}$ becomes empty. An example is illustrated in Fig. 5. For a triangle $p_{i} p_{w} p_{z}$ with $y\left(p_{w}\right)>y\left(p_{z}\right)$, we define the region $\tau\left(p_{i} p_{w} p_{z}\right)$ to be the intersection of the following three halfplanes: (i) $y>y\left(p_{z}\right)$; (ii) the halfplane lying below the line $p_{i} p_{w}$; and (iii) the halfplane bounded by the line $p_{w} p_{z}$ that does not contain $p_{i}$; see Fig. 6. Intuitively, this
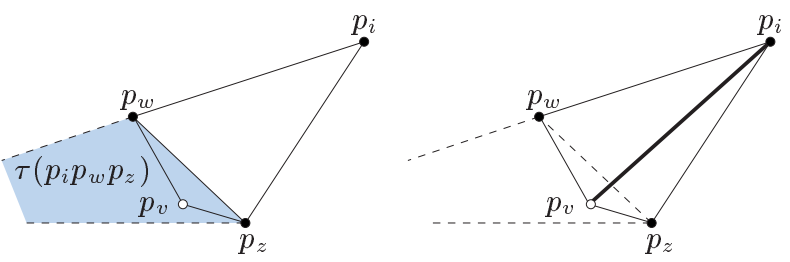

Fig. 6. If $p_{v}$ lies inside the shaded region $\tau\left(p_{i} p_{w} p_{z}\right)$, then an edge flip is performed. 
region $\tau\left(p_{i} p_{w} p_{z}\right)$ contains points that potentially should be visible to $p_{i}$ if edge $p_{w} p_{z}$ were not present.

(1) Remove the top triangle $p_{i} p_{w} p_{z}$ from $\mathcal{S}$. Assume that $y\left(p_{w}\right)>y\left(p_{z}\right)$.

(2) If $p_{w} p_{z}$ is an edge of the convex hull or $y\left(p_{z}\right)>y\left(p_{i}\right)$, go to step (1).

(3) Let $p_{v}$ be the other vertex adjacent to edge $p_{w} p_{z}$. If $p_{v} \notin \tau\left(p_{i} p_{w} p_{z}\right)$, go to step (1).

(4) Delete the edge $p_{w} p_{z}$, and insert the edge $p_{i} p_{v}$ (an edge flip).

(5) Push the triangle $p_{i} p_{v} p_{z}$ to $\mathcal{S}$.

(6) Push the triangle $p_{i} p_{w} p_{v}$ to $\mathcal{S}$.

By induction, one easily observes that the above algorithm constructs a valid triangulation $F$ within $\Delta^{+}$. Let $F^{-}$and $F^{+}$denote the fan triangulation within $\Delta^{+}$at time $t_{0}^{-}$and $t_{0}^{+}$, respectively.

Lemma 3.2. $\quad F$ as constructed by the above procedure is the same as $F^{+}$.

Proof. Observe that if an edge from $F$ or $F^{+}$does not have $p_{i}$ as an endpoint, then the edge is present in $F^{-}$as well, because the newly added edges in $F$ or $F^{+}$are all adjacent to $p_{i}$. Therefore, we only have to prove that the above procedure correctly identifies $\operatorname{Lk}\left(p_{i}\right)$ in $F^{+}$. Let $Q=\left\langle p_{k}=q_{0}, q_{1}, \ldots, q_{u}=p_{j}\right\rangle$ be the sequence of neighbors of $p_{i}$ in the resulting triangulation $F$. To prove the lemma, we need to show that (i) any $p_{i} q_{z}$ for $0 \leq z \leq u$ exists in $F^{+}$, and (ii) for any $p_{i} p_{z} \in F^{+}, p_{z} \in Q$. The first claim is easily shown by induction on the order in which the edges were added by the above procedure. At any time, the newly added edge is guaranteed to be present in $F^{+}$. We sketch the proof for the second claim below.

We prove the second claim by contradiction. By the first claim, $Q$ is a subset of $\operatorname{Lk}\left(p_{i}\right)$ at time $t_{0}^{+}$in $F^{+}$. Therefore, similar to $\operatorname{Lk}\left(p_{i}\right), Q$ consists of a convex chain $q_{0}, q_{1}, \ldots, q_{w}$, corresponding to the up neighbors of $p_{i}$ in $Q$, a $y$-monotone polygonal chain $q_{w+1}, \ldots, q_{u}$, corresponding to the down neighbors of $p_{i}$ in $Q$, and edge $q_{0} q_{u}$ (i.e., $p_{k} p_{j}$ ). See Fig. 7. Moreover, edges $p_{i} q_{0}, \ldots, p_{i} q_{u}$ are ordered in clockwise or counterclockwise order around $p_{i}$. Assume that $q_{s}$ and $q_{s+1}$ are two consecutive vertices of $Q$ such that there exists a point $q \in \operatorname{Lk}\left(p_{i}\right)$ lying between points $q_{s}$ and $q_{s+1}$ along $\operatorname{Lk}\left(p_{i}\right)$. Obviously, $q$ lies inside the wedge formed by $\overrightarrow{p_{i} q_{s}}$ and $\overrightarrow{p_{i} q_{s+1}}$, as $\operatorname{Lk}\left(p_{i}\right)$ is star-shaped. We now distinguish two cases, each of which leads to a contradiction:

1. $s+1 \leq w$. As $q_{0}, \ldots, q_{w}$ form a subset of a convex chain, triangle $p_{i} q_{s} q_{s+1}$ contains point $q$ (Fig. 7(a)). By construction, $p_{i} q_{s} q_{s+1}$ is a triangle in $F$. However, $F$ is a valid triangulation, so triangle $p_{i} q_{s} q_{s+1}$ cannot contain any of the input points, a contradiction.

2. $s+1>w$. Recall that $q$ lies in the wedge formed by the rays $\overrightarrow{p_{i} q_{s}}$ and $\overrightarrow{p_{i} q_{s+1}}$. If $q \in \Delta p_{i} q_{s} q_{s+1}$, then we arrive at the same contradiction as in case 1 (Fig. 7(b)). So $q$ lies in the halfplane bounded by $q_{s} q_{s+1}$ that does not contain $p_{i}$. Moreover, since $q_{s+1}$ lies on the $y$-monotone part of $\operatorname{Lk}\left(p_{i}\right)$ and $q$ lies between $q_{s}$ and $q_{s+1}$ along $\operatorname{Lk}\left(p_{i}\right)$, we have $y(q)>y\left(q_{s+1}\right)$, implying that $q \in \tau\left(p_{i} q_{s} q_{s+1}\right)$. As $q_{s}$ and $q_{s+1}$ are two consecutive neighbors of $p_{i}$ in $F, p_{i} q_{s} q_{s+1}$ must have been 


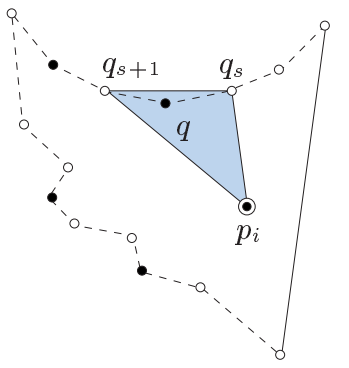

(a)

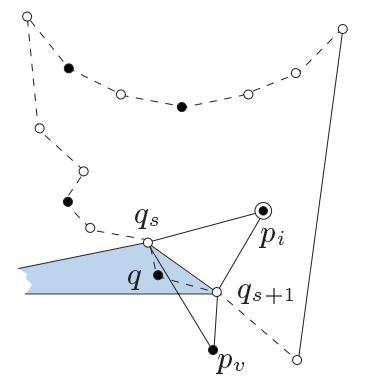

(c)

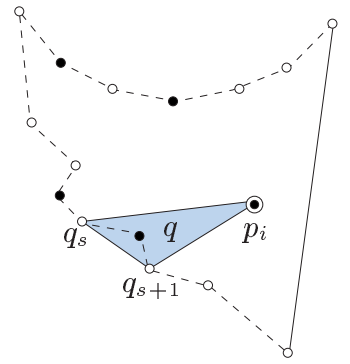

(b)

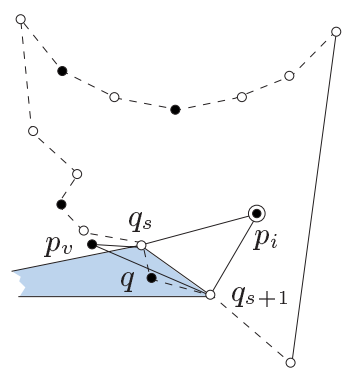

(d)

Fig. 7. Dashed polygon is $\operatorname{Lk}\left(p_{i}\right)$, with points denoted by hollow circles being vertices of $Q$. (a),(b) $q$ lies inside triangle $p_{i} q_{s} q_{s+1}$. (c),(d) $q$ lies in the region $\tau\left(p_{i} q_{s} q_{s+1}\right)$ (shaded), but triangle $p_{v} q_{s} q_{s+1}$ prevents vertex $q$ from being connected to $p_{i}$. All cases lead to a contradiction.

processed at some moment. The fact that $p_{i} q$ was not added at that time implies that there was another triangle, say, $p_{v} q_{s} q_{s+1} \in F^{-}$, incident upon edge $q_{s} q_{s+1}$, and $p_{v} \notin \tau\left(p_{i} q_{s} q_{s+1}\right)$. Notice that $q$ is not contained in either triangle $p_{v} q_{s} q_{s+1}$ or $p_{i} q_{s} q_{s+1}$. Now assume $p_{v}$ lies below $q_{s+1}$ (Fig. 7(c)). However, in this case, edge $p_{v} q_{s}$ could not have been present in $F^{-}$as it crosses edge $q q_{s+1}$ of $F^{-}$, but both points $q$ and $q_{s+1}$ are above $p_{v}$, a contradiction. The other case in which $p_{v}$ lies above line $p_{i} q_{s}$ can be handled similarly (Fig. 7(d)).

This proves the second claim, and the lemma follows.

The following lemma follows immediately from the above algorithm.

Lemma 3.3. The number of topological events induced by a crossing event of $p_{i}$ is proportional to the old degree plus the new degree of $p_{i}$.

Each topological event can be handled in $O(\log n)$ time, including the time spent in updating the global event queue. To detect all the three types of events, we can maintain the same three families of certificates as for a fan triangulation. 


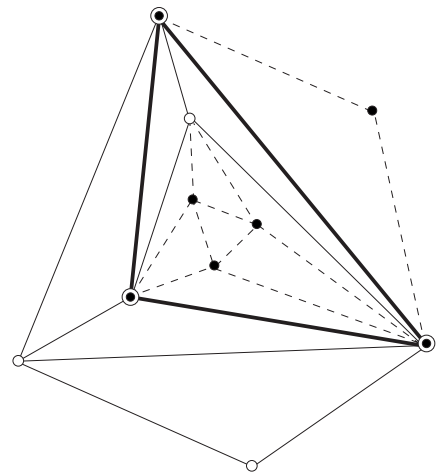

Fig. 8. A hierarchical fan triangulation with three levels-points in $R_{1}$ are denoted by double circles, in $R_{2} \backslash R_{1}$ by hollow circles, and in $R_{3} \backslash R_{2}$ by black circles.

\section{Hierarchical Fan Triangulation}

We now use the constrained fan triangulation to define a hierarchical fan triangulation $\mathbb{F}$. Let $\emptyset=R_{0} \subseteq R_{1} \subseteq \cdots \subseteq R_{w}=S$. Set $\mathbb{F}_{0}=\emptyset$, and for $i \geq 1$, define $\mathbb{F}_{i}=$ $\mathbb{F}\left(R_{i}, \mathbb{F}_{i-1}\right)$, i.e., $\mathbb{F}_{i}$ is the constrained fan triangulation of $R_{i}$ with respect to $\mathbb{F}_{i-1}$. By construction, $\mathbb{F}_{1}$ is the fan triangulation of $R_{1}$ and $\mathbb{F}_{i-1} \subseteq \mathbb{F}_{i}$. Set $\mathbb{F}=\mathbb{F}_{w}$. See Fig. 8 for an example.

Hereditary Event. As the points move, each $\mathbb{F}_{i}$ deforms continuously. The topology of $\mathbb{F}$ changes when there is an ordering, a visibility, or a crossing event in one of the $\mathbb{F}_{i}$ 's, and it can be processed as described in Sections 2 and 3. However, a topological change in $\mathbb{F}_{i}$ also propagates changes in $\mathbb{F}_{j}$ for $j>i$, as the insertion or deletion of an edge in $\mathbb{F}_{i}$ affects the visibility of points in $R_{j}$. We refer to an event in $\mathbb{F}_{i+1}$ caused by another event in $\mathbb{F}_{i}$, as a hereditary event of $\mathbb{F}_{i+1}$. We process hereditary events as follows. If $\operatorname{conv}\left(R_{i}\right)$ changes, i.e., a point $q \in R_{i}$ appears or disappears on $\operatorname{conv}\left(R_{i}\right)$, we update $\mathbb{F}_{i+1}$ within the exterior of $\operatorname{conv}\left(R_{i}\right)$ by inserting or deleting $q$ as described in the crossing event of Section 3. The number of topological events in this case is proportional to the new degree (if $q$ is inserted) or old degree (if $q$ is deleted) of $q$ within the exterior of $\operatorname{conv}\left(R_{i}\right)$. Moreover, if a triangle $\Delta$ in $\mathbb{F}_{i}$ is destroyed due to an edge insertion or deletion, we simply delete all the edges of $\mathbb{F}_{i+1}$ lying inside $\Delta$. As destroying a triangle unavoidably leads to creation of other triangle(s), we only describe how we perform reconstruction after creating a new triangle. Suppose a triangle $\Delta$ is created in $\mathbb{F}_{i}$, we reconstruct the affected portion of $\mathbb{F}_{i+1}$. More specifically, let $R_{i+1}^{\Delta}=R_{i+1} \cap \Delta$. We construct the fan triangulation $\mathbb{F}\left(R_{i+1}^{\Delta}\right)$ inside $\Delta$. The number of topological events in each newly created triangle $\Delta$ can be bounded by $O\left(\left|R_{i+1}^{\Delta}\right|\right)$. Each topological event induced by hereditary events can be handled in $O(\log n)$ time, including the time spent in updating the global event queue.

In the next section we analyze the performance of the hierarchical fan triangulation, assuming that $R_{i}$ is a random subset of $R_{i+1}$ of an appropriate size. 


\section{Analysis}

In the hierarchical fan triangulation $\mathbb{F}$ defined in Section 4 , we choose $R_{i-1}$ to be a random subset of $R_{i}$ of size

$$
\min \left\{\left|R_{i}\right|, 5\left|R_{i}\right|^{1-1 / i} \log \left|R_{i}\right|\right\}
$$

for $1<i \leq w$. Let $w=\lceil\sqrt{\log n / \log \log n}\rceil$. In this section we show that $\mathbb{F}$ has a near-quadratic number of topological changes as promised. To do this, we first focus on a specific level of the construction of $\mathbb{F}$. For notational convenience, let $P=R_{i}$, $R=R_{i-1}, \mathcal{T}(P)=\mathbb{F}_{i}, \mathcal{T}(R)=\mathbb{F}_{i-1}, n=|P|$, and $r=n^{1-1 / i}$, for some $1<i \leq w$. It follows that $\mathcal{T}(P)=\mathbb{F}(P, \mathcal{T}(R))$, and $|R|=\min \{n, 5 r \log n\}$.

Let $p, p_{1}, p_{2}, p_{3} \in P, t, t_{1}, t_{2} \in \mathbb{R}$, and $m \in \mathbb{N}$. Let $h\left(p_{1}, p_{2}\right)$ be the open halfplane to the left of the oriented line $\overrightarrow{p_{1} p_{2}}$. We define (see Fig. 9)

$$
\begin{aligned}
\left\langle p_{1}, p_{2}, p_{3} ; t\right\rangle & =\left\{p \in P \mid p(t) \in \Delta p_{1}(t) p_{2}(t) p_{3}(t)\right\}, \\
\left\langle p_{1}, p_{2} ; t_{1}, t_{2}\right\rangle_{s} & =\left\{p \in P \mid \exists t \in\left[t_{1}, t_{2}\right] \text { s.t., } p(t) \in p_{1}(t) p_{2}(t)\right\}, \\
\left\langle p_{1}, p_{2} ; t_{1}, t_{2}\right\rangle_{h} & =\left\{p \in P \mid \exists t \in\left[t_{1}, t_{2}\right] \text { s.t., } p(t) \in h\left(p_{1}(t), p_{2}(t)\right)\right\}, \\
\langle p ; m ; t\rangle_{\rho} & =\left\{\rho^{(k)}(p(t)) \mid 1 \leq k \leq m\right\}, \\
\langle p ; m ; t\rangle_{\gamma} & =\left\{\gamma^{(k)}(p(t)) \mid 1 \leq k \leq m\right\} .
\end{aligned}
$$

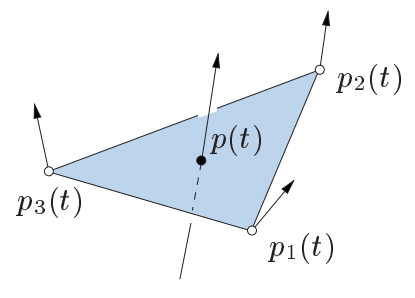

(a)

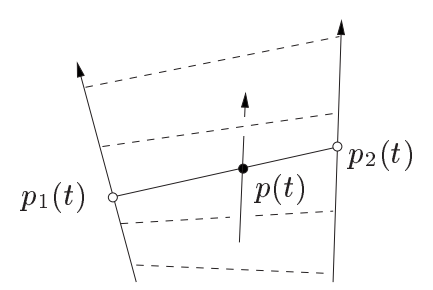

(b)

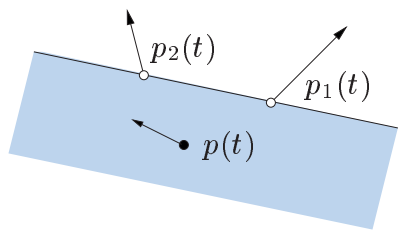

(c)

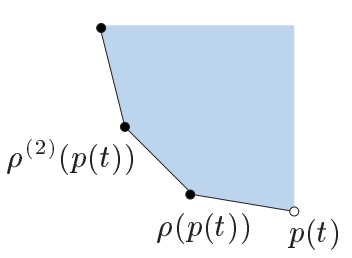

(d)

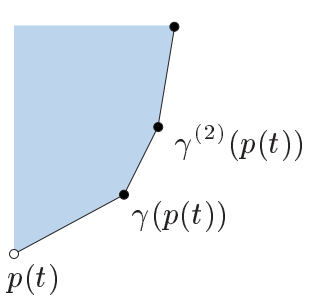

(e)

Fig. 9. Examples of different ranges: (a) $\left\langle p_{1}, p_{2}, p_{3} ; t\right\rangle$, (b) $\left\langle p_{1}, p_{2} ; t_{1}, t_{2}\right\rangle_{s}$, (c) $\left\langle p_{1}, p_{2} ; t_{1}, t_{2}\right\rangle_{h}$, (d) $\langle p ; m ; t\rangle_{\rho},(\mathrm{e})\langle p ; m ; t\rangle_{\gamma}$. 
We then let

$$
\begin{aligned}
& \mathcal{R}_{1}=\left\{\left\langle p_{1}, p_{2}, p_{3} ; t\right\rangle \mid p_{1}, p_{2}, p_{3} \in P, t \in \mathbb{R}\right\}, \\
& \mathcal{R}_{2}=\left\{\left\langle p_{1}, p_{2} ; t_{1}, t_{2}\right\rangle_{s} \mid p_{1}, p_{2} \in P, t_{1}, t_{2} \in \mathbb{R}\right\}, \\
& \mathcal{R}_{3}=\left\{\left\langle p_{1}, p_{2} ; t_{1}, t_{2}\right\rangle_{h} \mid p_{1}, p_{2} \in P, t_{1}, t_{2} \in \mathbb{R}\right\}, \\
& \mathcal{R}_{4}=\left\{\langle p ; m ; t\rangle_{\rho} \mid p \in P, m \in \mathbb{N}, t \in \mathbb{R}\right\}, \\
& \mathcal{R}_{5}=\left\{\langle p ; m ; t\rangle_{\gamma} \mid p \in P, m \in \mathbb{N}, t \in \mathbb{R}\right\} .
\end{aligned}
$$

Finally, let $\mathbb{X}=(P, \mathcal{R})$ be the range space with $\mathcal{R}=\bigcup_{1 \leq j \leq 5} \mathcal{R}_{j}$. We have the following lemma.

Lemma 5.1. $|\mathcal{R}|=O\left(n^{5}\right)$.

Proof. Consider three points $p_{1}, p_{2}$, and $p_{3}$ of $P$. As $t$ increases, $\left\langle p_{1}, p_{2}, p_{3} ; t\right\rangle$ changes only when some point $q \in P$ moves in or out of the triangle $p_{1} p_{2} p_{3}$. For any point $q$, this can only happen a constant number of times. Thus $\left\langle p_{1}, p_{2}, p_{3} ; t\right\rangle$ can change $O(n)$ times as $t$ goes from $-\infty$ to $+\infty$ for fixed $p_{1}, p_{2}$, and $p_{3}$. There are $O\left(n^{3}\right)$ different choices of $p_{1}, p_{2}, p_{3}$, implying that $\left|\mathcal{R}_{1}\right|=O\left(n^{4}\right)$. Similarly we can prove $\left|\mathcal{R}_{2}\right|=O\left(n^{4}\right)$ and $\left|\mathcal{R}_{3}\right|=O\left(n^{4}\right)$.

For any $p, q \in P$, if $y(p(t))<y(q(t))$, let $\theta(p(t), q(t))$ denote the angle formed by $\overrightarrow{p q}$ and the $+x$-direction at time $t ; \theta(p(t), q(t))$ is undefined at $t$ otherwise. The number of changes to $\rho(p)$ is the same as the complexity of the upper envelope of $\{\theta(p(t), q(t)) \mid q \neq p, q \in P\}$, which can be bounded by $\lambda_{s}(n)$ [16], where $\lambda_{s}(\cdot)$ is the maximum length of a Davenport-Schinzel sequence of order $s$. The value of $s$ depends on the maximum degree of polynomial of the trajectories of points in $P$; for example, $s=4$ if points move linearly. For fixed $p$ and $m$, as $t$ increases, $\langle p ; m ; t\rangle_{\rho}$ changes only when $\rho(q)$ changes for some $q \in P$. There are at most $n$ different choices for $p$ and $m$ each, thus we have $\left|\mathcal{R}_{4}\right|=O\left(n^{3} \lambda_{s}(n)\right)$, which is slightly larger than $O\left(n^{4}\right)$. Similarly $\left|\mathcal{R}_{5}\right|=O\left(n^{3} \lambda_{s}(n)\right)$. Putting everything together, $|\mathcal{R}|=O\left(n^{3} \lambda_{s}(n)\right)=O\left(n^{5}\right)$.

As time $t$ varies, the topological structure of $\mathcal{T}(P)$ may change: features such as edges or triangles may appear or disappear. The lifetime of a feature is the period between two of its consecutive appearances and disappearances. If an edge or a triangle occurs more than once, we count each occurrence as a different feature.

By (1), Lemma 5.1, and standard results from random sampling theory [15], $R$ is a $(1 / r)$-net of the range space $\mathbb{X}$ with high probability. Thus we obtain the following lemmas.

Lemma 5.2. With high probability:

(a) At any time, there are less than $n / r$ points of $P$ inside any triangle of $\mathcal{T}(R)$.

(b) Less than $n / r$ points of $P$ can cross any edge ever appearing in $\mathcal{T}(R)$ during its lifetime.

(c) Less than $n / r$ points of $P$ can ever appear in $h(p, q)$ during the period when $p, q \in R$ are two neighboring points on the convex hull of $R$ in clockwise order. 
Lemma 5.3. With high probability:

(a) For any $p \in P, m \in \mathbb{N}, t \in \mathbb{R}$,

$$
\left|\langle p ; m ; t\rangle_{\rho}\right| \leq\left(\left|\langle p ; m ; t\rangle_{\rho} \cap R\right|+1\right) \cdot n / r .
$$

(b) For any $p \in P, m \in \mathbb{N}, t \in \mathbb{R}$,

$$
\left|\langle p ; m ; t\rangle_{\gamma}\right| \leq\left(\left|\langle p ; m ; t\rangle_{\gamma} \cap R\right|+1\right) \cdot n / r .
$$

Proof. Suppose that

$$
\langle p ; m ; t\rangle_{\rho} \cap R=\left\{\rho^{\left(i_{1}\right)}(p(t)), \rho^{\left(i_{2}\right)}(p(t)), \ldots, \rho^{\left(i_{v}\right)}(p(t))\right\},
$$

where $1 \leq i_{1}<i_{2}<\cdots<i_{v} \leq m$. Let $i_{0}=0$ and $i_{v+1}=m+1$. We have

$$
\begin{aligned}
\langle p ; m ; t\rangle_{\rho}= & \left(\bigcup_{j=1}^{v+1}\left\langle\rho^{\left(i_{j-1}\right)}(p(t)) ; i_{j}-i_{j-1}-1 ; t\right\rangle_{\rho}\right) \\
& \cup\left\{\rho^{\left(i_{1}\right)}(p(t)), \rho^{\left(i_{2}\right)}(p(t)), \ldots, \rho^{\left(i_{v}\right)}(p(t))\right\} .
\end{aligned}
$$

Moreover, by the properties of a $(1 / r)$-net of $\mathbb{X}$, we know that with high probability,

$$
\left|\left\langle\rho^{\left(i_{j-1}\right)}(p(t)) ; i_{j}-i_{j-1}-1 ; t\right\rangle_{\rho}\right| \leq n / r-1,
$$

for $1 \leq j \leq v+1$. We thus obtain

$$
\left|\langle p ; m ; t\rangle_{\rho}\right| \leq(v+1) \cdot(n / r-1)+v \leq(v+1) \cdot n / r .
$$

We can prove (b) in a similar manner.

Let $\operatorname{deg}(p(t))$ denote the degree of point $p \in P$ in $\mathcal{T}(P)$ at time $t$.

Lemma 5.4. If a point $p \in P$ lies on an edge of $\mathcal{T}(R)$ at time $t_{0}$, then both $\operatorname{deg}\left(p\left(t_{0}^{-}\right)\right)$ and $\operatorname{deg}\left(p\left(t_{0}^{+}\right)\right)$are bounded by $n / r$ with high probability.

Proof. If $p\left(t_{0}^{-}\right)$or $p\left(t_{0}^{+}\right)$lies in the interior of $\operatorname{conv}(R)$, then we have $\operatorname{deg}\left(p\left(t_{0}^{-}\right)\right) \leq n / r$ or $\operatorname{deg}\left(p\left(t_{0}^{+}\right)\right) \leq n / r$ with high probability by Lemma 5.2(a). If $p$ crosses an edge $p_{1} p_{2}$ of $\partial \operatorname{conv}(R)$ at $t_{0}$, say, from inside to outside, then at $t_{0}^{+}$, all points adjacent to $p$ lie in the open halfplane bounded by $p_{1} p_{2}$ that is disjoint from $\operatorname{conv}(R)$. It then follows from Lemma 5.2(c) that $\operatorname{deg}\left(p\left(t_{0}^{+}\right)\right) \leq n / r$. A symmetric argument shows $\operatorname{deg}\left(p\left(t_{0}^{-}\right)\right) \leq n / r$ if $p$ moves from the exterior to the interior of $\operatorname{conv}(R)$ at $t_{0}$.

For a set $A \subseteq P$ of points, let $\psi(A)$ denote the total number of topological changes to $\mathcal{T}(A)$ over time. We will bound $\psi(P)$ in terms of $\psi(R)$, where $R$ is a $(1 / r)$-net with respect to the range space $\mathbb{X}$. For simplicity, we omit the phrase "with high probability." We now bound the number of topological changes induced by each type of event as discussed in Section 3 and 4. 
Hereditary Events. Each hereditary event either causes us to re-triangulate $\mathcal{T}(P)$ inside $O$ (1) triangles of $\mathcal{T}(R)$, or insert or delete a point of $R$ that appears or disappears on $\partial \operatorname{conv}(R)$. By Lemma 5.2(a) and 5.4, each such event induces $O(n / r)$ topological changes. Since each hereditary event is caused by a topological event of $\mathcal{T}(R)$, the total number of topological changes induced by the hereditary events is

$$
O(n / r) \cdot \psi(R)
$$

Crossing Events. By Lemma 5.2(b), at most $n / r$ points cross an edge of $\mathcal{T}(R)$ during its lifetime. By Lemmas 3.3 and 5.4, each such event induces $O(n / r)$ topological changes. Since there are $O(\psi(R)+|R|)$ distinct edges in $\mathcal{T}(R)$ over the entire history, the total number of topological changes induced by crossing events is

$$
O((n / r) \cdot(n / r) \cdot(\psi(R)+|R|))=O\left(n^{2} / r^{2}\right) \cdot \psi(R) .
$$

Visibility Events. Recall that a visibility event occurs when there is a change in $\rho(p)$ or $\gamma(p)$ with respect to $\Delta \in \mathcal{T}(R)$ for some point $p \in P_{\Delta}$ (recall $P_{\Delta}$ is the set of points of $P$ inside $\Delta$ ). As discussed earlier, each such event induces a constant number of topological changes. Therefore, we only need to bound the number of visibility events.

A visibility event occurs due to the collinearity of $p$ and two other points $q$ and $z$, where $p, q$, and $z$ lie within the same face, say $\Delta$, of $\mathcal{T}(R)$. If $\Delta$ is a triangle in $\mathcal{T}(R)$ (i.e., $p \in \operatorname{conv}(R))$, then by Lemma 5.2, only $O(n / r)$ points can ever appear in $\Delta$ during its lifetime. Using a lower-envelope argument as in the proof of Lemma 5.1, it can be shown that the number of visibility events happening to $p$ while $p \in \Delta$ is $O\left(\lambda_{s}(n / r)\right)$. Since there are $O(\psi(R)+|R|)$ triangles in $\mathcal{T}(R)$ over the entire history, the total number of visibility events inside $\operatorname{conv}(R)$ is

$$
O\left((n / r) \cdot \lambda_{s}(n / r) \cdot(\psi(R)+|R|)\right)=O\left(n^{2} \log (n) / r^{2}\right) \cdot \psi(R) .
$$

Next, suppose a point $p \notin \operatorname{conv}(R)$ causes a visibility event. Let $l_{\mathrm{t}}\left(\right.$ resp. $l_{\mathrm{b}}$ ) be the horizontal line passing through the highest (resp. lowest) point of $R$. We distinguish three cases: (a) $p$ lies between $l_{\mathrm{t}}$ and $l_{\mathrm{b}}$ and to the left (resp. right) of conv $(R)$, and $\gamma(p)$ (resp. $\rho(p)$ ) changes; (b) $p$ lies between $l_{\mathrm{t}}$ and $l_{\mathrm{b}}$ and to the left (resp. right) of conv $(R)$, and $\rho(p)$ (resp. $\gamma(p))$ changes; and (c) $p$ lies above $l_{\mathrm{t}}$ or below $l_{\mathrm{b}}$.

It is easy to observe that cases (b) and (c) at $t=t_{0}$ correspond to a change in

$$
\arg \max _{q \in P} \theta(p(t), q(t)) \quad \text { or } \quad \arg \min _{q \in P} \theta(p(t), q(t))
$$

at time $t_{0}$. Therefore, there are an $O\left(n \lambda_{s}(n)\right)=O\left(n^{2} \log n\right)$ number of such events, by a lower-envelope argument similar to the one in the proof of Lemma 5.1.

Now consider case (a) (depicted in Fig. 10). Assume without loss of generality that $p$ lies to the left of $\operatorname{conv}(R)$. Let $p_{1} p_{2}$ be the edge on $\operatorname{conv}(R)$ that is hit by the ray emanating from $p$ towards its right (assuming $p_{1}, p_{2}$ are in clockwise order). Observe that $q, z$, the two points that are involved in the visibility event with $p$, must lie in the triangle $p p_{1} p_{2}$, and therefore $p, q, z \in h\left(p_{1}, p_{2}\right)$. Thus, during the period when $p_{1}, p_{2}$ are two neighboring points on $\operatorname{conv}(R)$ in clockwise order, the number of events that three points of $P$ in $h\left(p_{1}, p_{2}\right)$ become collinear is $O\left(n^{3} / r^{3}\right)$, by Lemma 5.2(c). If 


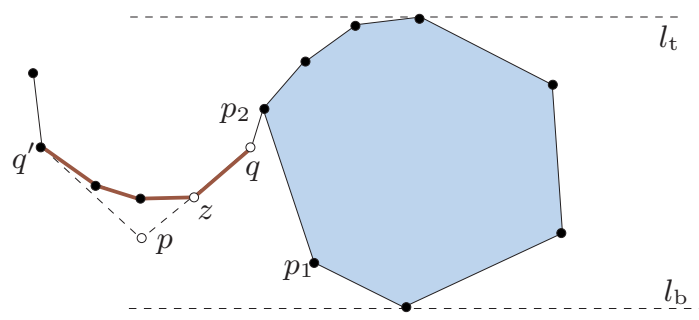

Fig. 10. The shaded region is $\operatorname{conv}(R)$, and the thick chain is $\mathcal{V}(p)$.

points move along algebraic trajectories, the combinatorial structure of $\operatorname{conv}(R)$ changes $O\left(|R| \lambda_{s}(|R|)\right)=O\left(|R|^{2} \log n\right)$ times [16]. Therefore there are $O\left(n^{3}|R|^{2} \log (n) / r^{3}\right)$ such events.

In total, the number of topological changes to the triangulation of $P$ caused by visibility events is bounded by

$$
O\left(n^{3}|R|^{2} \log (n) / r^{3}+n^{2} \log n\right)+O\left(n^{2} \log (n) / r^{2}\right) \cdot \psi(R) .
$$

Ordering Events. The number of topological changes induced by an ordering event at time $t_{0}$ when the $y$-coordinates of two mutually visible points $p$ and $q$ become equal is proportional to $\left|\mathcal{V}_{p q}\left(t_{0}\right)\right|$, i.e., the number of points that are visible from both $p$ and $q$ at time $t_{0}$. If $p$ and $q$ lie inside a triangle face of $\mathcal{T}(R)$, then $\left|\mathcal{V}_{p q}\left(t_{0}\right)\right|=O(n / r)$. Since there are $O\left(n^{2}\right)$ ordering events, the total number of topological changes induced by such events is $O\left(n^{3} / r\right)$. Now assume that $p$ and $q$ lie in the exterior of $\operatorname{conv}(R)$, and let $I$ be the set of such events. Obviously, $|I| \leq n^{2}$. Denote by $m_{i}$ the number of points of $R$ on $V_{p q}\left(t_{0}\right)$ for the $i$ th such event from $I$. By Lemma 5.3, the total number of topological changes caused by this type of event is at most

$$
\sum_{i}\left(m_{i}+2\right) n / r=O\left(n^{3} / r\right)+(n / r) \cdot \sum_{i} m_{i}
$$

Lemma 5.5. $\quad \sum_{i \leq|I|} m_{i}=O\left(n^{2}|R|^{2} \log (n) / r^{2}\right)$.

Proof. Suppose two points $p$ and $q$ lying outside $\operatorname{conv}(R)$ induce an ordering event at time $t_{0}$, i.e., $y\left(p\left(t_{0}\right)\right)=y\left(q\left(t_{0}\right)\right)$ and $p$ is visible from $q$ at $t_{0}$. Let $z_{1}, z, z_{2} \in R$ be three consecutive points on $\partial \operatorname{conv}(R)$ in clockwise order at $t_{0}$ and $z \in V_{p q}\left(t_{0}\right)$. Recall that $h\left(z_{1}, z\right)$ is the open halfplane to the left of $\overrightarrow{z_{1}} z$. We have that $p, q \in h\left(z_{1}, z\right) \cup h\left(z, z_{2}\right)$ as $z$ is visible to both $p$ and $q$. For fixed edges $z_{1} z$ and $z z_{2}$ of $\partial \operatorname{conv}(R), z$ can only be involved in $O\left(n^{2} / r^{2}\right)$ such events because, by Lemma 5.2(c), only $O(n / r)$ points ever appear in $h\left(z_{1}, z\right)$ (resp. $h\left(z, z_{2}\right)$ ) during the lifetime of edge $z_{1} z$ (resp. $z z_{2}$ ). Let $u_{z}$ be the total number of times that $z$ is involved in such an event. As points in $R$ move, $\operatorname{conv}(R)$ may change $O\left(|R| \lambda_{s}(|R|)\right)=O\left(|R|^{2} \log n\right)$ times [16], therefore the number of distinct triples of consecutive vertices on $\partial \operatorname{conv}(R)$ is $O\left(|R|^{2} \log n\right)$. We then have

$$
\sum_{z \in R} u_{z}=O\left(n^{2}|R|^{2} \log (n) / r^{2}\right) .
$$


Using a simple double counting argument, we obtain that $\sum_{i \leq|I|} m_{i}=\sum_{z \in R} u_{z}$. This proves the lemma.

It follows from the above lemma that the total number of topological changes induced by ordering events is bounded by

$$
O\left(n^{3} / r+n^{3}|R|^{2} \log (n) / r^{3}\right)=O\left(n^{3}|R|^{2} \log (n) / r^{3}\right) .
$$

Summing (2)-(5) and substituting the values of $|R|$ and $r$, we obtain the following recurrence for $\psi(P)$ :

$$
\psi(P) \leq O\left(n^{2+1 / i} \log ^{3} n\right)+O\left(n^{2 / i} \log n\right) \cdot \psi(R) .
$$

Returning to the hierarchical fan triangulation, let $n_{i}$ be the size of $R_{i}$, then

$$
\psi\left(n_{i}\right) \leq c_{1} n_{i}^{2 / i} \log \left(n_{i}\right) \psi\left(n_{i-1}\right)+c_{2} n_{i}^{2+1 / i} \log ^{3} n_{i},
$$

where $c_{1}, c_{2}>0$ are constants, and

$$
n_{i-1}=\left|R_{i-1}\right|=\min \left\{n_{i}, 5 n_{i}^{1-1 / i} \log n_{i}\right\} .
$$

It can be verified by induction that the solution to the above recurrence is

$$
\psi\left(n_{i}\right) \leq n_{i}^{2+1 / i} \log ^{3 i}\left(n_{i}\right) 2^{c i},
$$

where $c$ is a sufficiently large constant. In particular, for $i=w=\lceil\sqrt{\log n / \log \log n}\rceil$, i.e., the hierarchical fan triangulation, we have

$$
\psi(n)=n^{2} 2^{O(\sqrt{\log n \cdot \log \log n})} .
$$

We conclude with the following main result.

Theorem 5.6. Let $S$ be a set of $n$ points moving in $\mathbb{R}^{2}$. If the motion of $S$ is algebraic, a triangulation of $S$ can be maintained by a randomized algorithm so that the number of topological events processed by the algorithm is $n^{2} 2^{O(\sqrt{\log n \cdot \log \log n)}}$ with high probability, and each topological event requires $O(\log n)$ time.

As a special case of our problem, we consider a scenario in which only $k$ out of $n$ points are moving. By extending our previous technique, we can show that there exists a triangulation whose number of topological changes is roughly $O(n k)$. We describe this triangulation briefly.

The overall framework follows that for the case with $n$ moving points. The major difference is that, at each level of the hierarchical fan triangulation, instead of sampling the points all at once, we sample the static and moving points separately. For any point set $P$, let $P^{s} \subseteq P$ be the set of static points in $P$, and let $P^{t} \subseteq P$ be the set of moving points in $P$.

Set $w=\lceil\sqrt{\log k / \log \log n}\rceil$. Let $\emptyset=R_{0} \subseteq R_{1} \subseteq \cdots \subseteq R_{w}=S$, such that for $1<i \leq w, R_{i-1}^{s}$ is a random subset of $R_{i}^{s}$ of size

$$
\min \left\{\left|R_{i}^{s}\right|, O\left(\left|R_{i}\right| \cdot\left|R_{i}^{t}\right|^{-1 / i} \log \left|R_{i}^{s}\right|\right)\right\},
$$


and $R_{i-1}^{t}$ is a random subset of $R_{i}^{t}$ of size

$$
\min \left\{\left|R_{i}^{t}\right|, O\left(\left|R_{i}^{t}\right|^{1-1 / i} \log \left|R_{i}^{t}\right|\right)\right\} .
$$

We construct the hierarchical fan triangulation $\mathbb{F}$ of $S$ as described in Section 4.

Let $n_{i}=\left|R_{i}\right|$ and $k_{i}=\left|R_{i}^{t}\right|$, for $1 \leq i \leq w$. By the same method as in [2], one can show that the fan triangulation $\mathbb{F}_{1}$ changes $O\left(k_{1}^{4 / 3} \lambda_{d}\left(n_{1}\right)\right)=O\left(n_{1} k_{1}^{4 / 3} \log n_{1}\right)$ times. Using this fact, and by similar analysis as above, it can be shown that the number of topological changes to $\mathbb{F}_{i}$ is bounded by $n_{i} k_{i}^{1+1 / i} \log ^{3 i}\left(n_{i}\right) 2^{c i}$ for some constant $c$, for $1 \leq i \leq w$. In particular, the number of topological changes to the hierarchical fan triangulation is $n k \cdot 2^{O(\sqrt{\log k \cdot \log \log n})}$. Without going into the detail, we conclude with the following theorem.

Theorem 5.7. Let $S$ be a set of $n$ points in $\mathbb{R}^{2}$, with $k$ of them moving with algebraic trajectories of bounded degree. A triangulation of $S$ can be maintained by a randomized algorithm so that the number of topological events processed by the algorithm is $n k$. $2^{O(\sqrt{\log k \cdot \log \log n)}}$ with high probability, and each topological event requires $O(\log n)$ time.

\section{Conclusions}

In this paper we have described a randomized algorithm for maintaining a triangulation of a set of moving points in $\mathbb{R}^{2}$. If the motion is algebraic, then the expected number of topological events is $n^{2} 2^{O(\sqrt{\log n \cdot \log \log n})}$. Our result almost matches the $\Omega\left(n^{2}\right)$ lower bound, and improves over the previously best known result [2] by nearly a factor of $n^{1 / 3}$. If only $k$ points of the point set are moving, the expected number of topological changes reduces to $n k \cdot 2^{O(\sqrt{\log k \cdot \log \log n})}$.

Although the triangulation that we maintain is conceptually simple, it is not clear how to derandomize the algorithm efficiently. It will be interesting to find a simple deterministic triangulation, easy to implement, and with a near-quadratic number of topological changes. A major open problem in this area is, of course, bounding the number of topological events in the Delaunay triangulation of a set of moving points. The best known upper bound is near cubic while the best known lower bound is quadratic [6].

Another interesting problem is whether Steiner points can help to reduce the complexity of kinetic triangulations. Agarwal et al. [1] showed that the $\Omega\left(n^{2}\right)$ lower bound on the total number of topological changes to a triangulation still holds even if $O(n)$ Steiner points are allowed and can move along any continuous trajectories. However, we expect that with the introduction of Steiner points, a much simpler triangulation with a near-quadratic number of topological changes may be found.

\section{Acknowledgments}

We thank Herbert Edelsbrunner, Leo Guibas, Alper Üngör, and the two anonymous referees for their helpful comments. 


\section{References}

1. P. K. Agarwal, J. Basch, M. de Berg, L. J. Guibas, and J. Hershberger, Lower bounds for kinetic planar subdivisions, Discrete Comput. Geom., 24 (2000), 721-733.

2. P. K. Agarwal, J. Basch, L. J. Guibas, J. Hershberger, and L. Zhang, Deformable free space tiling for kinetic collision detection, Internat. J. Robotics Res., 21 (2002), 179-197.

3. P. K. Agarwal et al., Algorithmic issues in modeling motion, ACM Comput. Surveys, 24 (2002), 550-572.

4. P. K. Agarwal, L. J. Guibas, J. Hershberg, and E. Veach, Maintaining the extent of a moving point set, Discrete Comput. Geom., 26 (2001), 253-274.

5. P. K. Agarwal and C. M. Procopiuc, Advances in indexing mobile objects, IEEE Bull. Data Engr., 25 (2002), 25-34.

6. G. Albers, L. J. Guibas, J. Mitchell, and T. Roos, Voronoi diagrams of moving points, Internat. J. Comput. Geom. Appl., 8 (1998), 365-380.

7. M. Atallah, Some dynamic computational geometry problems, Comput. Math. Appl., 11 (1985), 11711181.

8. J. Basch, L. J. Guibas, and J. Hershberger, Data structures for mobile data, J. Algorithms, 31 (1999), 1-28.

9. M. W. Bern and D. Eppstein, Mesh generation and optimal triangulation, in: Computing in Euclidean Geometry (D.-Z. Du and F. K.-M. Hwang, eds.), 2nd edition, number 4 in Lecture Notes Series on Computing, World Scientific, Singapore, 1995, pp. 47-123.

10. L. P. Chew, Near-quadratic bounds for the $L_{1}$ Voronoi diagram of moving points, Comput. Geom. Theory Appl., 7 (1997), 73-80.

11. J. Donea, Arbitrary Lagrangian-Eulerian finite element methods, in: Computational Methods for Transient Analysis (T. B. Belytschk and T. J. R. Hughes, eds.), North-Holland, Elsevier, Amsterdam, 1983, pp. 474516.

12. H. Edelsbrunner, Triangulations and meshes in computational geometry, Acta Numer., 9 (2000), 133-213.

13. J. Erickson, D. Guoy, J. Sullivan, and A. Üngör, Building space-time meshes over arbitrary spatial domains, Proc. 11th Internat. Meshing Roundtable, 2002, pp. 391-402.

14. L. J. Guibas, Kinetic data structures: a state of the art report, in: Robotics: The Algorithmic Perspective (P. K. Agarwal, L. Kavraki, and M. Mason, eds.), A. K. Peters, Natick, MA, 1998, pp. 191-209.

15. D. Haussler and E. Welzl, $\varepsilon$-Nets and simplex range queries, Discrete Comput. Geom., 2 (1987), 127-151.

16. M. Sharir and P. K. Agarwal, Davenport-Schinzel Sequences and Their Geometric Applications, Cambridge University Press, New York, 1995.

Received July 27, 2004, and in revised form June 30, 2005. Online publication September 11, 2006. 